\title{
Cost Analysis of WDM and TDM Fiber-to-the-Home (FTTH) Networks: A System-of-Systems Approach
}

\author{
Theodore Rokkas, Ioannis Neokosmidis, Dimitris Katsianis, and Dimitris Varoutas, Senior Member, IEEE
}

\begin{abstract}
A system-of-systems (SoS) approach for wavelengthdivision multiplexing (WDM) and time-division multiplexing (TDM) fiber-to-the-home (FTTH) telecommunication networks is presented. Cost evolution curves for individual systems as well for whole FTTH WDM and TDM networks are presented. The analysis can be exploited for a fast and accurate analysis of FTTH deployment costs in dense urban, urban, and suburban areas from the technoeconomic point of view, which is of paramount importance for telecom operators, equipment vendors, regulators, and policy makers. The impact of delaying the deployments or adopting different rollout strategies is also investigated and presented. The SoS emergent behavior is further revealed using exploratory modeling. The results reveal that in all cases, the WDM solution is more expensive than TDM. The total cost for suburban areas is almost six times higher than in dense urban areas and four times than urban areas.
\end{abstract}

Index Terms - Cost learning curves, fiber-to-the-home (FTTH), optical communications, system-of-systems (SoS), technoeconomics, telecommunications, time-division multiplexing (TDM) SoS, wavelength-division multiplexing (WDM) SoS.

\section{INTRODUCTION}

$\mathbf{O}$ VER the past few years, a trend in society and mainly in industry for system integration is observed. Illustrative examples are the aerospace and defense industry. However, for many industries, the effort for integration and holistic system analysis and design constitutes a key part in drawing up their business strategies. This trend mainly comes from consumers' need for systems that provide advanced features. This effort, which is known as "system of systems" (SoS), is an emerging and multidisciplinary research area.

Although the concept of SoS is not new, it has gained increased attention in the last decade. This research area is still in embryonic stage which is also illustrated by the lack of a precise definition [1], [2]. An SoS can be described as a set or arrangement of interdependent systems that are related or connected to provide a given capability [3]. Furthermore, the components of the SoS are themselves autonomous embedded complex systems

Manuscript received January 23, 2011; revised September 12, 2011, March 3, 2012, and August 12, 2012; accepted October 28, 2012. Date of current version December 17, 2012. This paper was recommended by Associate Editor J. Chen.

The authors are with the Technoeconomic Group of Optical Communications and Broadband Access Laboratory, Faculty of Informatics and Telecommunications, School of Science, University of Athens, GR15784 Athens, Greece (e-mail: trokkas@di.uoa.gr; i.neokosmidis@di.uoa.gr; dkats@di.uoa.gr; d.varoutas@di.uoa.gr).

Color versions of one or more of the figures in this paper are available online at http://ieeexplore.ieee.org.

Digital Object Identifier 10.1109/TSMCC.2012.2227999 that can be diverse in technology, context, operation, geography, and conceptual frame [4].

System-of-Systems Engineering (SoSE) is a field that deals with the complexity and problems of SoS. Reference to SoSE began in the late 1990s. An important barrier to this effort is the lack of specific and general approaches and methods to address the practical difficulties in SoS. Unfortunately, traditional systems engineering is not applicable in the case of SoS mainly because they have not been developed to address high levels of ambiguity and uncertainty [4].

The present state of SoSE can be divided into two paths. The first mainly deals with technical issues (interoperability, net-centricity, and integration) [5], while the second is dominated by concerns with human/social, contextual, and higher level inquiry to produce purposeful responses to complex system problems [4]. The above separation reveals the multidimensional nature of these problems. It is then evident that the main goal of the various research teams, that is to develop unified SoSE techniques and generate solutions in a formalized, repeatable manner, can only be accomplished by incorporating epistemological, methodological, and ontological aspects. It should be noted that the latter gives to SoSE a multidisciplinary character [6]. However, the degree to which, and how, multiple disciplines can be combined must be investigated.

The design and construction of a fiber-to-the-home (FTTH) network rests on both paths of the SoSE. On the one side, issues such as marketing, demand, and adoption of the network are related to the social and humanitarian nature of the problems of the FTTH network. On the other hand, the construction, dimensioning, operation, and maintenance of the network deal with technical issues such as selection, integration, and interoperability of the various components of the network.

However, to the best of authors' knowledge, the design, manufacture, and provision of a telecommunication network such as of the FTTH networks have not attracted the interest of SoSE experts and have not yet been studied in the light of an SoS, although such networks meet the aforementioned criteria. So far in the literature, various networks and sets have been investigated in the form of SoS. However, these studies mainly concerns maritime transportation systems [7], disaster and damage situations [8]-[10], defense systems [11], [12], global earth observation systems [13], health systems [14], etc.

Due to the increasing interest on the FTTH networks and their deployment strategies, a new approach incorporating all aspects of network development, administration, and operation is required. To this end, an SoS approach is proposed, aiming to exploit the benefits of SoS methodologies in this critical issue for the development of telecommunications. This methodology 
is valuable because it helps basically decision makers to perceive notions of underlying costs associated with technical, economic, and even social issues and thus, derive strategies for resource allocations, and infrastructure investments using concepts such as emergent behaviors of the users and interdependences between the complex system integrations required [15].

In order to implement the proposed methodology, appropriate models and tools have been developed. The application of traditional technoeconomic models [16]-[18] in such a complex environment would lead to time-consuming procedures, and in many cases, it is difficult to be interpreted by nonexperts. However, advanced models can enhance the design of such systems with valuable information improving the process and minimizing the risk of being consumed at inappropriate options. In this perspective, SoS engineering methodologies and tools can be more than valuable to such complex engineering issues as those arisen from FTTH debate.

In detail, in this paper, the notion of SoS in telecommunications and especially in FTTH networks is introduced. Metrics associated with first installation costs in the case of a greenfield operator as well as the incremental cost for the completion of the FTTH SoS deployment are provided. Multiple paths providing numerous possible $\mathrm{SoS}$ realizations are given through exploratory modeling [19], [20].

The remainder of this paper is organized as follows. In Section II-A, the SoS nature of a passive optical network $(\mathrm{PON})$ is depicted. Topology and area characteristics are given in Section II-B. The system under consideration and the cost model are presented in Sections II-C and II-D, respectively. In Section III, learning curves for telecommunication components are illustrated. The results obtained by the cost analysis of the SoS are illustrated in Section IV. Concluding remarks are provided in Section V.

\section{SySTEM-OF-SySteMS APPROACH FOR FIBER-TO-THE-HOME TIME-Division MULTIPLEXING AND WAVELENGTH-DIVISION MULTIPLEXING NETWORKS}

\section{A. System-of-Systems Approach for Fiber-to-the-Home}

Using the definitions that have been proposed by various researchers in the past, a set of systems can be characterized as an "SoS" if it fulfills the following features [21].

1) An SoS involves the integration of multiple, potentially previously independent, systems into a higher level system (metasystem) to perform a mission/purpose for which each member plays an integral role.

2) An SoS generates capabilities beyond which any of the constituent member (sub) systems is independently capable of producing.

3) Integration into an SoS evokes some degree of constraint (surrender of autonomy) for previously independent systems.

4) An SoS is a complex system and as such exhibits dynamic and emergent behavior, is difficult to grasp, and is problematic to engineer.

5) An $\mathrm{SoS}$ is characterized by the geographical distribution of its component systems.

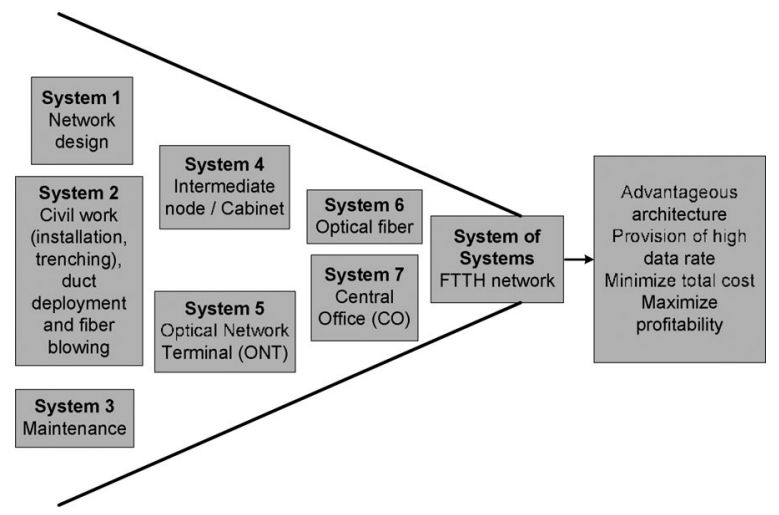

Fig. 1. SoS approach of an FTTH network.

The increased complexity of SoS is mainly due to the capability of the constituent systems to operate independently. Hence, apart from cooperating, the systems could also compete for subtasks. Another factor leading to the complexity is the inability to predict the operation of an SoS since the latter is usually occurred in uncertain environments. Finally, the SoS is a dynamic entity as new systems are added and current systems are replaced or removed.

Because of these unique features, the development of suitable methods is required to study an $\mathrm{SoS}$ and predict its eventual behavior as the individual systems change, causing changes in the larger SoS. Several methods such as agent-based modeling [22], metasynthesis [23], and systemigrams [24] have been proposed in the literature. In fact, the study of complex problems under the SoS perspective is equivalent to finding an equilibrium point between the individual systems characteristics. In this paper, the investigation of time-domain multiplexing (TDM) and wavelength-domain multiplexing (WDM) technologies adoption under different policies as well as exploratory modeling have been proposed as tools to study the SoS emergent characteristics. Numerical evaluations of model outcomes across a large set of possible SoS representations are performed giving the "whole picture" of SoS in a time sequence of different periods.

To begin with, one should initially identify the constituent systems of the SoS under consideration. In fact, the whole process of designing, constructing, and maintaining an FTTH network consists of many independent components that are combined to provide very high data rate in low cost to the end user, as shown in Fig. 1. These components can be characterized as systems mainly due to their increased complexity as well as their nonlinear behavior and their operation in uncertain environments. In order to reveal the system nature of the components, the structure of the Central Office (CO) is described as a representative example.

As shown in Fig. 2, the $\mathrm{CO}$ is a complex system because of its dimensions, that is, the large number of heterogeneous elements that comprise it. Specifically, under the term CO, one can incorporate the air condition which ensures the proper operation of the devices, the power supply unit (PSU), batteries for uninterrupted operation of the $\mathrm{CO}$, the switches, the optical 


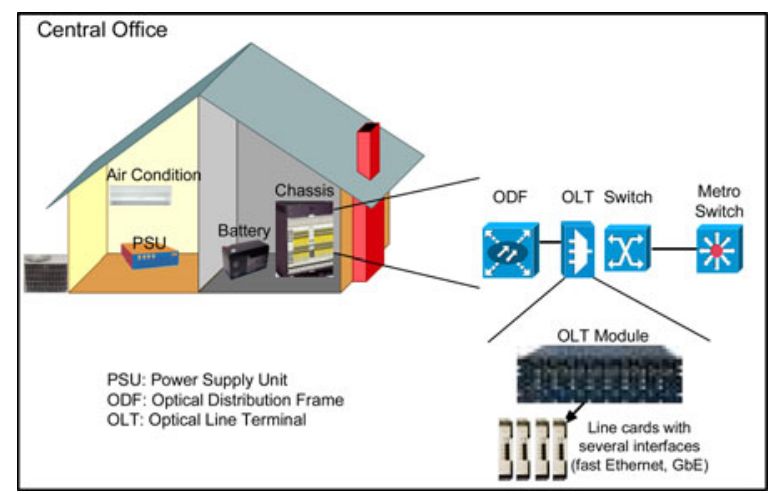

Fig. 2. System nature of the CO.

distribution frame (ODF), and the optical line terminal (OLT). The key element of the CO is the OLT, which is the endpoint of the service provider of a PON. Furthermore, one should also add the abundance of line cards that can be installed in the OLT corresponding to different functions and network interfaces. Finally, the aforementioned devices are integrated into the $\mathrm{CO}$ in order to connect the backbone network to the access network and thus provide services to the end users. However, integration and interoperability of these elements is not easy, taking into account the complexity of these elements, the fast developments in these technologies, as well as legacy systems and backwards compatibility requirements. In the same sense, the reader can easily expand the system nature of each part of illustrated in Fig. 1.

It should be noted that a communication medium is of great importance for the proper operation of a next-generation network. In fact, such a medium is required so that the components of the SoS can collaborate and communicate with each other. Without the existence of a communication medium, which is an optical fiber in the case of a PON, the study of the SoS would not be feasible. As this medium of communication has interfaces with each of the individual systems, it clearly plays a key role in their operation affecting the performance of the whole SoS.

Before proceeding in such an analysis, the question whether the design, construction, and maintenance of an FTTH network is in fact an SoS should be answered. Hence, one should investigate if the design, construction, and maintenance of an FTTH network meet the characteristics of an SoS, as defined in the literature.

It should be noted that each of the constituent systems plays its own significant role in the provision of high data rates. Simultaneously, an FTTH network is constructed in order to supply advanced services that would not be available from each of the constituent systems. On the other hand, the integration of different parts of an FTTH network in an SoS structure restricts their characteristics. The optical fiber which is one of the key parts of the network is designed to support very long distances. However, in an FTTH network, the optical fiber is essentially forced to be limited to the short distances of the network coverage. Additionally, in the design, manufacture, and provision of such a network, as well as during its operation various factors such as selection of appropriate components and interfaces, geographical and morphological characteristics as well as demand should be taken into account. The multitude of these factors increases the complexity of the system imposing the involvement of experts apart from engineers.

Another feature of an SoS is the differences in geographic location of the member systems [15], [25]. It is worth noting that this is a common characteristic of PONs, where the distances between the $\mathrm{CO}$ and the intermediate node as well as between the intermediate node and the end users are few tens of kilometers. The information is transmitted from one system to another providing the desired services to end users. This feature is further enhanced by the construction and maintenance parts of the proposed SoS. It is interesting to note that both can perform either centralized of distributed operations. For example, during the maintenance process, several problems can be addressed centrally from the $\mathrm{CO}$ using proper software platforms. On the other hand, more serious problems such as damages should be addressed in the field by several workshops which are usually independent and physically dispersed.

At the same time, the uncertainty of demand and price evolution, as well as the variety of architectures suitable for an FTTH network, enhances the SoS nature of this network. In the case of an access network, the network operator constructs a system to meet the requirements of users in order to minimize the total cost of the network and, therefore, to maximize profitability. The problem of building such a network is mainly an optimization problem that includes variables like geographical distribution in addition to the cost of various components, the cost of wiring, the installation cost, as well as the cost of maintenance.

By incorporating in the system design the variety of network architectures and technologies that can be selected, as well as the uncertainty regarding the demand, the SoS character of the problem is enhanced. The solution to this nonlinear problem can highlight the advantageous architecture for the network under consideration, facilitating the decision making process of the network operator.

It is widely accepted that the construction, dimensioning, operation, and maintenance of the FTTH network deal with technical issues such as selection, integration, and interoperability of the various components and systems of the network deployment and operation. In addition, social and economic factors such as cost of capital, marketing, demand forecast, and epidemic adoption are also related to the FTTH services and strategies. Therefore, it can be concluded that an FTTH network rests on both paths of the SoSE and such a notion can be used in order to provide useful insights about the complexity of network systems deployment and operations. In the following paragraphs, this notion is used in order to provide such insights about the technoeconomic aspects of the SoS FTTH network, by calculating cost figures which can be exploited by telecom operators, vendors, regulators, and policy makers.

\section{B. Topology and Area Characteristics}

In this study, three areas are considered and described in terms of subscribers' density, loop lengths, and geographical and 
TABLE I

AREA CHARACTERISTICS

\begin{tabular}{|c|c|c|c|}
\hline Area type & Dense Urban & Urban & Suburban \\
\hline Central Office & 1 & 1 & 1 \\
\hline Cabinets & 256 & 256 & 512 \\
\hline Number of buildings & 1024 & 2048 & 16384 \\
\hline Subscribers per building & 64 & 32 & 4 \\
\hline Total potential subscribers & 65536 & 65536 & 65536 \\
\hline Total Service area $\left(\mathrm{km}^{2}\right)$ & 12 & 32 & 160 \\
\hline Density $\mathrm{d}_{\mathrm{s}}$ (subs $\left./ \mathrm{km}^{2}\right)$ & 5461 & 2048 & 410 \\
\hline Duct availability (Main Network) & $0 \%$ & $0 \%$ & $0 \%$ \\
\hline
\end{tabular}

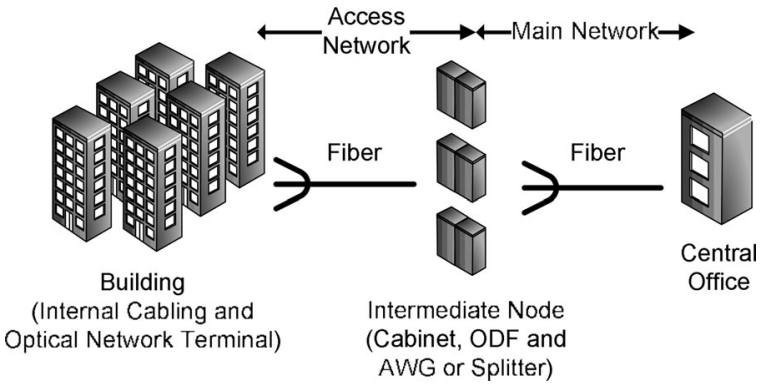

Fig. 3. Network topology.

market characteristics. The areas under investigation, dense urban, urban, and suburban, respectively, are presented at Table I.

A geometric model similar to that in [17] is used in order to calculate the lengths of cables and ducts in the network. In addition, 65536 possible customers and no duct availability are assumed in each area which is served by a $\mathrm{CO}$ connected to the cabinets through dedicated fibers. The customers are divided into groups of 32, and each group is served by a power splitter (in the case of TDM-PON) or an arrayed-waveguide grating (AWG) (in the case of WDM-PON) installed at the cabinet. It should be noted that in an FTTH scenario, each user is connected to the cabinet via its own fiber. The network topology is presented in Fig. 3.

\section{Passive Optical Network Architectures Under Consideration}

In PON architectures, a shared fiber is used to connect the $\mathrm{CO}$ with the intermediate node, and the users are connected to the intermediate node with dedicated fibers of short lengths. In this case, there is no active equipment between the $\mathrm{CO}$ and the user. There are two alternative PON architectures: the TDM-PON and the WDM-PON that are illustrated in Fig. 4(a) and (b), respectively. In the TDM-PON scenario, a single wavelength is used to connect all the users to the $\mathrm{CO}$ and a different time slot is allocated to each user. The users are connected to the CO through a simple power splitter. In the downlink direction, data are broadcasted from the OLTs over a single wavelength channel which can be shared on a frame basis using the Ethernet protocol. In the uplink, another wavelength channel is used to carry upstream data transmitted by the optical network terminals (ONTs). The same wavelength is used for all ONTs in the PON. TDM can be used to allocate time slots on which every (a)

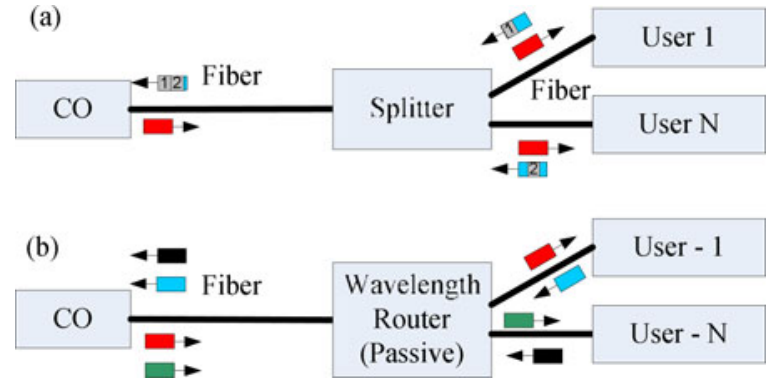

Fig. 4. PON architecture: (a) TDM-PON and (b) WDM-PON.

user can transmit its own data destined to the CO. This architecture has the advantage of being simple to implement. However, the splitter introduces splitting losses which scale linearly with $1 / N, N$ being the number of users [26].

In the WDM-PON scenario, to each user, a different wavelength is assigned and a multiwavelength source (WDM bank) is placed at the CO. In order to distribute the wavelengths to the users, a static wavelength router such as an AWG is used at the cabinet location [26]. The device's cyclic spectral properties enable a single AWG to route the downstream and upstream wavelengths simultaneously.

The difference between the two solutions is in the optoelectronic equipment that is used in the $\mathrm{CO}$, the intermediate node, and the user premises. In the case of WDM, this equipment is more expensive because of the more advanced optical components that must be installed.

\section{Cost and Maintenance Calculations}

The network operator is usually responsible for constructing and maintaining the network. However, the costing approaches and methodologies used in telecommunications are a topic of great debate due to the regulation obligations arisen by competition rules. Within this framework of regulations, the incremental costing was developed, based on specific assumptions and factors. The existing methodologies are based on top-down or bottom-up approaches with specific time windows in which the costs have to be calculated and sustained. In addition, interactions with the existing installed network for the capacity to be added should also be taken into account, which is, in general, a difficult issue to be resolved [27], [28]. Regulatory obligations are often related to efficiency, which incurs that operator should provide service using the least-cost methods taking into account prices and technology. Since these costs are not easily measureable, proxy models are often used but these models are also questionable, due to the fact that they are lacking of accuracy in calculating existing cost-efficient access lengths and future-proof network deployment since demand is evolving in a fast but still uncertain way [29].

In this perspective, the SoS approach can offer the approaches and the methods to deal with costing methods by incorporating existing and forecasting data in the costing approach. In the following paragraphs, this approach will be developed and used. The approach is based on the Long-Rum Incremental Cost (LRIC) methodology, which is the most known costing 
TABLE II

MAINTENANCE MATERIAL ClasSiFICATIONS

\begin{tabular}{|c|c|}
\hline Maintenance Class & Maintenance Cost percentage $R_{\text {class }}$ \\
\hline Sites and enclosures & $1.0 \%$ \\
\hline Optical components & $3.0 \%$ \\
\hline Fiber cables & $0.5 \%$ \\
\hline Electronics & $2.0 \%$ \\
\hline
\end{tabular}

approach in telecommunications investments. The methodology aims in a long-run time window to measure the change in the total costs of the telecom operator that arises from an increase or decrease in the telecom output by a substantial and discrete increment considering, in the long-run, all costs variable. It should be denoted that the use of incremental methodology for cost analysis should be carefully considered since it tolerates strong assumptions, which need to be usually evaluated and eventually restated [30], [31]. In addition, as the cost of bandwidth is nowadays lower than the cost of maintaining and operating the network, the measurement of Operation, Administration, and Maintenance cost is not a trivial issue since several nontypical factors are significant. In [32], some of these factors such as technology mix and capacity usage have been discussed in the case of US telecom industry.

However, the LRIC method, enhanced with the SoSE concept, may overcome the shortcomings of existing approaches and provide useful information about the actual costs of telecommunication networks. For the analysis presented hereafter, it has been assumed that no infrastructure is present in the areas under investigation. This represents a "Greenfield" case, which can be exploited from policy makers, regulators, and new entrants in telecom market as a proxy model for decision making. It can also be used to calculate state-aid subsidization or to benchmark cross-subsidies between elements and services.

In order to estimate the required number of network components throughout the study period, broadband access forecasts are carried out according to the methodology described in [33]. For each component as will be described in Section III, a price curve is calculated representing the component's cost throughout the study period. The maintenance costs for the total network are estimated from the cost of each of its constitutive parts based on the appropriate architectural model. The maintenance $\operatorname{cost} M_{i}$ for each of the installed network elements is calculated according to equation:

$$
M_{i}=\frac{V_{i-1}+V_{i}}{2}\left(P_{i} \cdot R_{\text {class }}+P_{\text {labor }} \frac{\text { MTTR }}{\mathrm{MTBR}}\right)
$$

where $P_{\text {labor }}$ is the cost of the work hour (in $€$ /hour), and MTBR and MTTR stand for mean time between repairs (in years) and mean time to repair (in hours) for each cost component, respectively. $V_{i}$ is the equipment volume in year $i, P_{i}$ is the price of cost item in year $i$, and $R_{\text {class }}$ is the maintenance cost percentage (defined by choosing maintenance material class for every cost component as per Table II).

In more detail, the maintenance cost is comprised with two cost elements. The term $P_{i} \cdot R_{\text {class }}$ reflects an annual fixed cost over component cost which is irrelevant to component failures. This cost item might be charged by vendors or not. The second term in parenthesis $P_{\text {labour }} \frac{\mathrm{MTTR}}{\mathrm{MTBR}}$ reflects the annual equivalent cost in case of failures or unavailability. The cost items should be summed up over the total numbers of components/subsystems available in the network. However, since this number is changing, the average number of specific components over the past two years is used instead.

The cost of the whole system as presented later on is calculated by summing up all the individual costs plus the maintenance costs for all the required subsystems.

\section{E. Demand Model}

A four-parameter logistic model [33], [34] was used to make broadband subscription forecasts. These models are recommended and used for long-term forecasts even in light of the introduction of new services. The aggregated demand for subscriptions is given by [33]

$$
Y_{t}=\frac{M}{\left(1+e^{\alpha+\beta t}\right)^{\gamma}}
$$

where $Y_{t}$ is the demand (subscriptions) at time $t, M$ is the saturation level or total market potential, and $\alpha, \beta$, and $\gamma$ are parameters, which can be estimated by a regression analysis using historical data of existing broadband connections.

\section{LEARNING CURVES FOR TELECOM COMPONENTS}

The rollout of an FTTH network includes several investments in specific telecommunications components used in order to provide end-to-end connections with fiber. These components are often new in the market, and the majority of them have been invented and constructed specifically in order to provide FTTH solutions. The industry behind these equipments is quite new, and the cost evolution of them follows the evolution of the telecommunication market and is related to particular learning curves.

Learning curves are used in the industry to predict reduction in production time or production cost as a function of produced volume. The causes of cost reductions are better control of the production process, new production methods, new technology, redesign of the product, standardization, and automatization.

Learning curves present the relationship between cost (or time) and level of activity on the basis of the effect of learning. An early study by Wright [35] disclosed the "80\% learning" effect, which indicates that a given operation is subject to a $20 \%$ productivity improvement each time the activity level or production volume doubles.

Learning curve can serve as a predictive tool to obtain time estimates for tasks that are repeated within a project life cycle. Several alternate models of learning curves have been presented in the literature. Some of the classical models are as follows [36]:

1) Log-linear model;

2) $S$-curve model;

3) Stanford-B model;

4) DeJong's learning formula;

5) Levy's adaptation function;

6) Glover's learning formula; 
TABLE III

VARIATION IN $n_{r}(0)$ AND $\Delta T$ FOR EACH VOLUME CLASS

\begin{tabular}{|c|c|c|c|}
\hline Volume class & $\mathbf{n}_{\mathbf{r}}(\mathbf{0})$ & $\boldsymbol{\Delta} \mathbf{T}$ & Example \\
\hline Old Fast & 0.5 & 5 & Fiber termination \\
\hline Mature Medium & 0.1 & 10 & Fiber cables \\
\hline New Medium & 0.01 & 10 & Switches \\
\hline New Slow & 0.01 & 20 & Lasers \\
\hline Emerging Medium & 0.001 & 10 & $\begin{array}{c}\text { WDM/TDM } \\
\text { components }\end{array}$ \\
\hline Straight line & 0.1 & 1000 & Cable installation \\
\hline
\end{tabular}

7) Pegels' exponential function;

8) Knecht's upturn model;

9) Yelle's product model.

The basic log-linear model is the most popular learning curve model. It expresses a dependent variable (e.g., production cost) in terms of some independent variable (e.g., cumulative production).

In some later work [37] which is related to telecommunication components, Wright and Crawford's learning curve [35], [38] models for cost predictions were examined. The models for cost predictions were extended not only to estimate the costs as a function of number of produced units, but also as a function of time. The cost prediction of each network component is described by expansion of the learning curve given as a function of the several parameters. Therefore, the price $P(t)$ of each network element is assumed to follow the extended learning curve [37] :

$$
P(t)=P(0)\left[n_{r}(0)^{-1}\left\{1+e^{\ln \left[n_{r}(0)^{-1}-1\right]-\frac{2 \ln 9}{\Delta T} t}\right\}^{-1}\right]^{\log _{2} K}
$$

where $P(0)$ is the price in the reference year $0, n_{r}(0)$ is the relative accumulated production volume in year 0 , (units sold in this year), $\Delta T$ is the time for the total production volume to grow from $10 \%$ to $90 \%$ of its maximum value, and $K$ is the learning curve coefficient and reflects the price reduction experienced when the production volume is doubled.

In cases where historical data are available, the parameters $K$ and $\Delta T$ can be determined using a standard regression analysis.

In the analysis presented hereafter, the parameters of the learning curve are stored in a database built within the technoeconomic tool, which contains more than 1000 different network components [30], [39]. The components and subsystems are grouped in several volume classes. Example values used for the various volume classes are shown in Table III. In the same way, the $K$ parameter is estimated based on type of component or subsystem, reflecting the learning process from other similar components or systems. Fig. 5 presents the effect of $\Delta T$ for a given $n_{r}(0)$, while Fig. 6 presents the effect of $n_{r}(0)$ for a given $\Delta T$ for a component with initially cost of $1000 €$.

In the cost database, all components and subsystems are listed with a given $n_{r}(0), \Delta T$, and $K$ value in addition to the estimated cost $P(0)$ in year 0 . Then, the extended learning curve is uniquely defined and the prediction of the costs is determined.

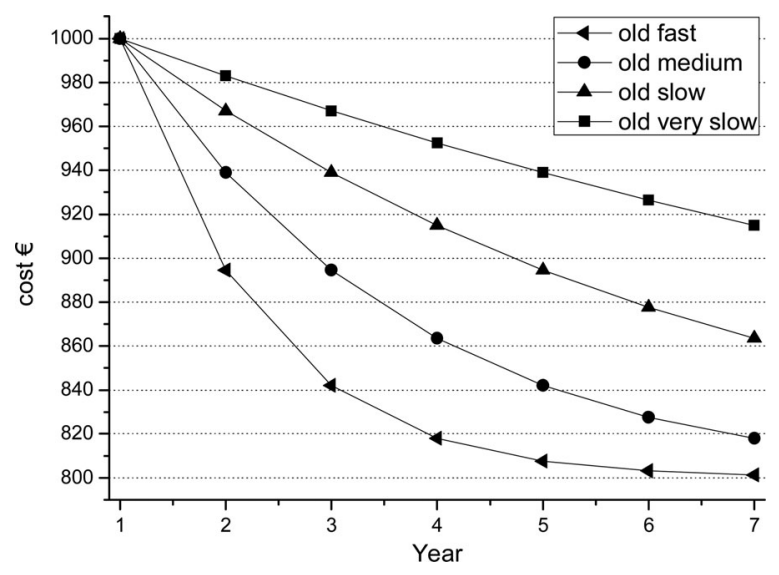

Fig. 5. Effect of $\Delta \tau$ for given $n_{r}(0)=0.5$ for a component with initially cost $1000 €$.

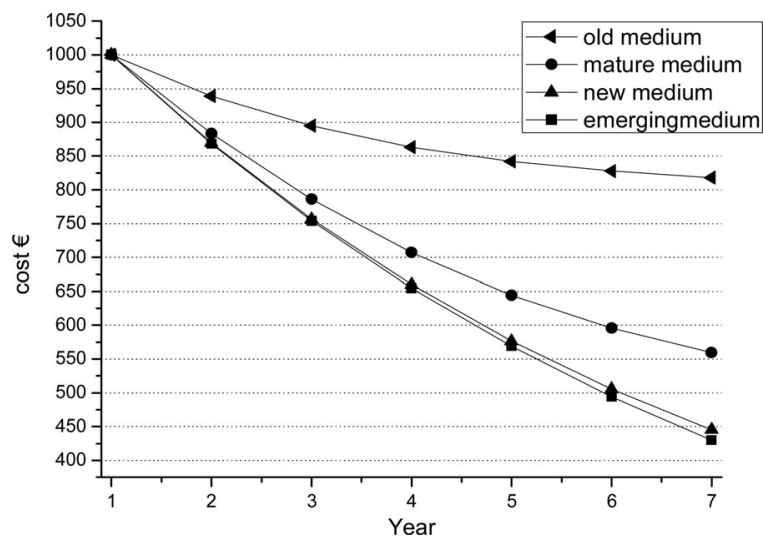

Fig. 6. Effect of $n_{r}(0)$ for given $\Delta \tau=10$ for a component with initially cost $1000 €$.

In addition, the volume classes are chosen to cover the two aspects of cost components: the type and maturity of cost components. The definition Old, Mature, etc., stands for the years that the specific components offered in the market. The definition Fast, Medium, etc., stands for time in years to grow the total production volume from $10 \%$ to $90 \%$ of its maximum value. For example, for the components used in the analysis presented herein, the installation or civil work costs are part of the straight line class, fiber cables costs are in the mature medium class, lasers are new slow class components, switches belong to the new medium class and new optoelectronic devices used at the PON architectures under study are emerging medium class components.

In case the components are new and no historical costs exist, a priori values have to be chosen. Examples are shown in Tables III and IV. Typical values of the learning curve coefficient are from 1 (100\%) (Meaning no cost reduction) to 0.7 (70\%), giving $30 \%$ reduction for doubling of production volume. An additional doubling of the production will reduce the cost by $51 \%$.

Fig. 7 illustrates the curve prices for some of network key components normalized at $1000 €$ initial cost. It should be noted 
TABLE IV

$K$ Values for Component Groups

\begin{tabular}{|l|c|}
\hline \multicolumn{1}{|c|}{ Component group } & K value \\
\hline Civil work & 1 \\
\hline Copper & 1 \\
\hline Installation & 1 \\
\hline Sites and enterprises & 0.95 \\
\hline Fiber & 0.9 \\
\hline Electronics & 0.8 \\
\hline Advanced optical components & 0.7 \\
\hline
\end{tabular}

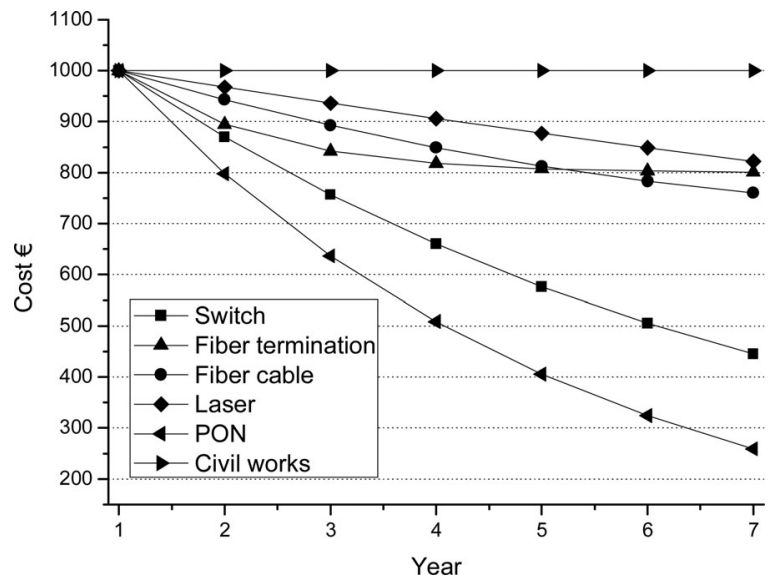

Fig. 7. Cost curves for some components used normalized at initially cost of $1000 €$.

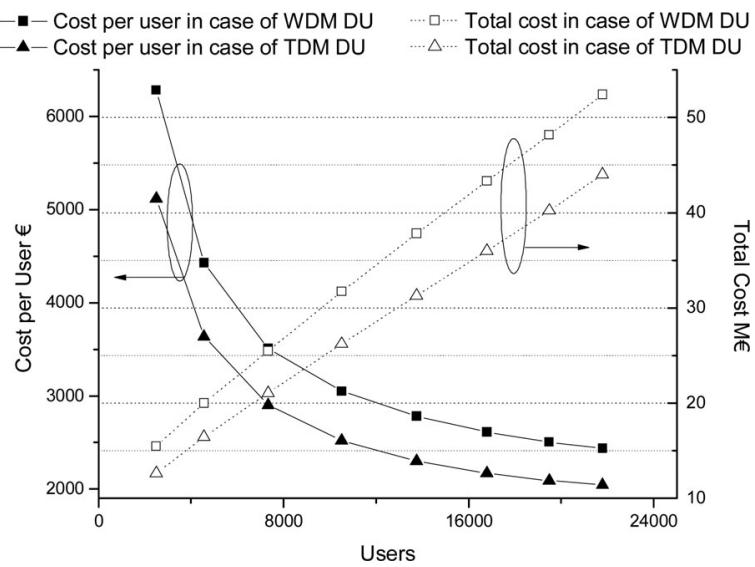

Fig. 8. SoS incremental cost of WDM and TDM for dense urban area.

that the actual cost prices for the components used in the study are mostly different than those presented in Fig. 7.

\section{RESULTS AND DISCUSSION}

\section{A. System-of-Systems Incremental Cost}

Fig. 8 illustrates the SoS incremental cost (cost per user and the total cost of the network installation) for both TDM and WDM PON technologies for the dense urban area. The WDM solution appears more expensive than TDM due to the most expensive equipment used at customer premises and network.

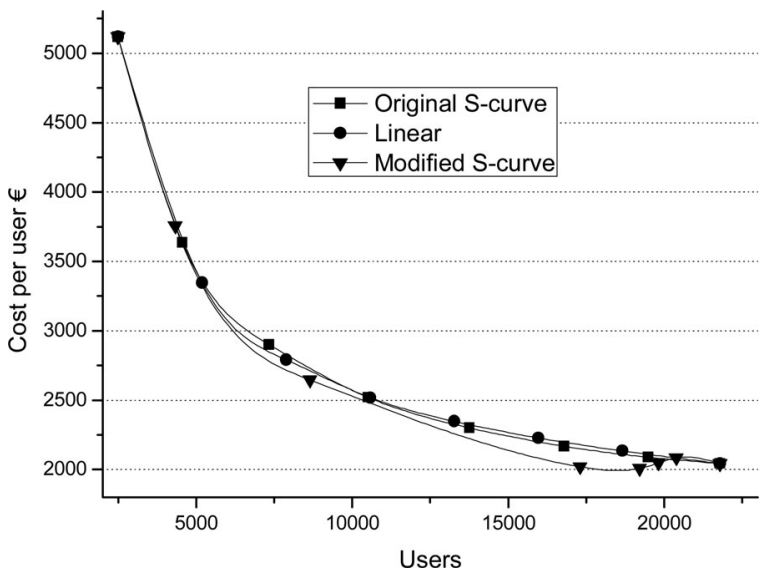

Fig. 9. Cost per user for various demand models in the case of TDM.

The cost per user is initially high due to large investments and low initial utilization of the network. As the rollout of the FTTH network is proceeding and more customers are connected to the network, the SoS incremental cost (per user) reduces yielding in the area of $2000 €$ and $2500 €$ for TDM and WDM solutions, respectively. The shape of the cost per user function is similar to price curves figures illustrating the additional cost for the implementation of the FTTH SoS.

In order to estimate the impact of user distribution and adoption in terms of homes connected throughout the years to the cost per user, the demand has been modeled using three different ways: the original $S$-curve described in (2), the slight modified $S$-curve with the saturation point $M$ reached before the end of the study period and a simplified linear one. All three models have the same initial and end values, and the difference among them is that in the linear model, more subscribers are connected earlier in the FTTH period that the two other ones. The original model described in (2) can be seen as a typical intermediate model in contradiction to the other two alternatives. Fig. 9 illustrates the cost per user that is calculated for the case of TDM in dense urban areas for all three demand models.

It can be observed that there are no significant differences by using three different demand models other than that the $S$-curve model that reaches saturation earlier has slightly lower cost prices. In this case, it can be concluded that an increased rate as in the $S$-curve model leads to a reduction in the connection cost per user by $10 \%$. This result can be exploited by policy makers and regulators aiming to boost FTTH deployment by subsidizing the deployment cost. However, for the rest of the analysis, the original model for the demand has been used as described in (2). This is due to the fact that it reflects a rather typical technology adoption curve, and it is generic enough to reflect the different adoption strategies worldwide.

Fig. 10 illustrates the SoS incremental cost (cost per user and total cost) for both alternative PON technologies for all three examined areas. The solid lines present the total costs (right $Y$-axis), while the dotted ones the cost per user (left $Y$-axis). The solid dots represent WDM and the open ones TDM, while the square, triangle, and circle symbols represent dense urban, urban, and suburban areas, respectively. In all areas, 


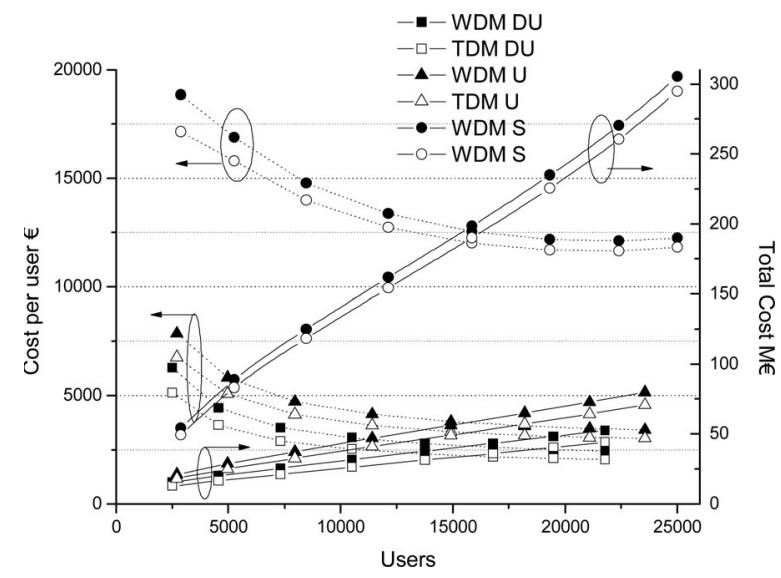

Fig. 10. Cost per user and total cost of WDM and TDM for all examined areas.

WDM is more expensive than TDM, and in suburban areas, the costs are significantly higher than dense urban and urban areas due to the lower subscriber's density leading to higher duct and fiber length deployments that are the major part of the investments. The SoS incremental cost (per user) reaches almost $12500 €$, while in the urban areas, the cost is below $3500 €$ (dense urban 2000€). The total SoS cost for the suburban areas is around six times larger than in dense urban areas and around four times from urban. This explains the operators willingness to announce investment plans for FTTH in dense urban areas, and the discussion follows about the public state aid, in terms of regulation measures or subsidization procedures for the rest of the areas [40].

\section{B. Time-Division Multiplexing and Wavelength-Division Multiplexing Adoption Under Different Policies}

Nowadays, several FTTH projects have been announced especially in Europe [41], but the vast majority have been delayed. The impact of delaying the WDM deployment in a dense urban area from one to three years have been studied and presented hereafter in order to incorporate this decision alternative in the analysis. For simplicity, it has been assumed that no other alternative networks are implemented in the same area. Fig. 11 reveals that the SoS cost per user varies significantly when the operator suspends the introduction of FTTH for one year: the cost per user reduces to almost $35 \%$, while the delay for two and three years leads to a $23 \%$ and $16 \%$ reduction, respectively, for the WDM solution. For the case of TDM, the reduction is $30 \%$ for one year delay, and $21 \%$ and $14 \%$ for two and three years, respectively. This variance is due to the fact that the operators are benefitted from the increasing demand in these areas and the cost reduction of the individual components and subsystems.

Fig. 12 illustrates the total cumulative investments cost for WDM and TDM cases (dense urban area) for different FTTH launch year. The delay in the rollout leads to a reduction of about $1 \mathrm{M} €$ per year for WDM and $0.4 \mathrm{M} €$ for TDM of suspension due to the reduced price of the necessary components and systems, as described in Section III. Furthermore, the operator can benefit from the time value of the money (distribution of the

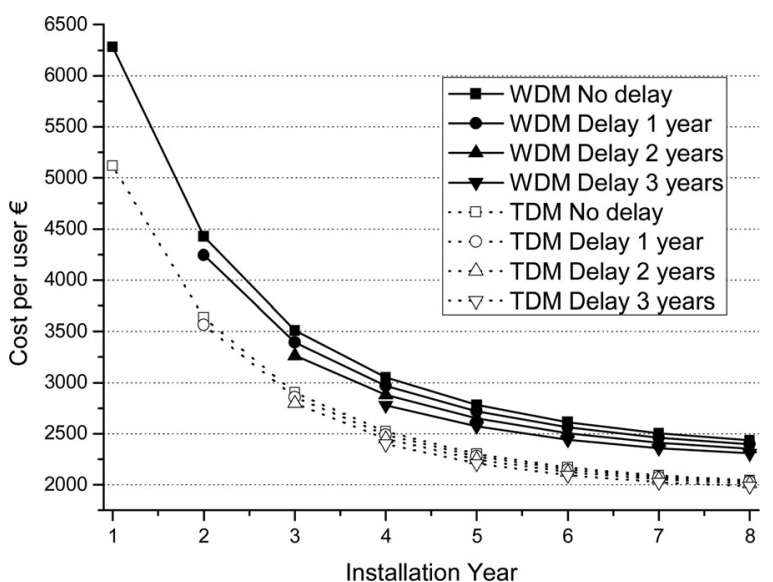

Fig. 11. Impact of delay at cost per user for TDM and WDM in dense urban areas.

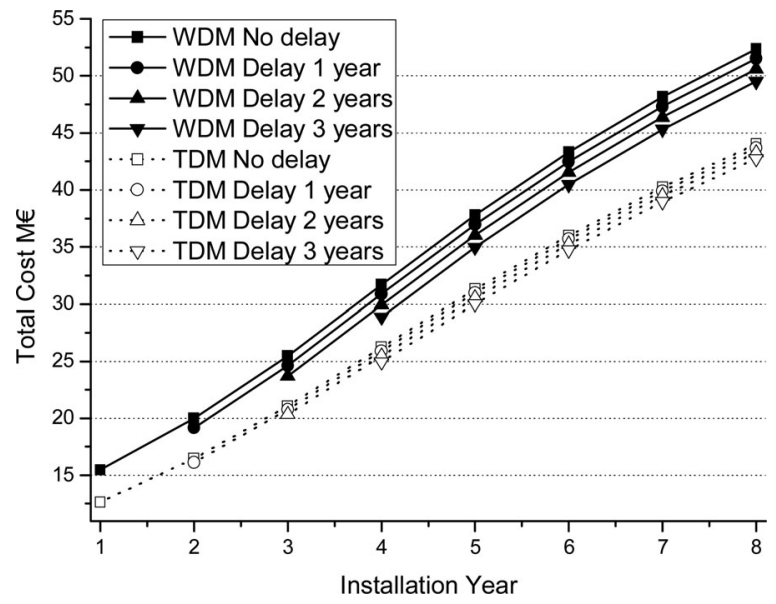

Fig. 12. Impact of delay at total cost for TDM and WDM in dense urban areas.

costs within the rollout). For example, the total cost for covering the expected subscriber with no delay in the rollout of the project reaches $32 \mathrm{M} €$ for the first four years $\left(\mathrm{Y}_{0}: 15.5 \mathrm{M} €, \mathrm{Y}_{1}\right.$ : $4.5 \mathrm{M} €, \mathrm{Y}_{2}: 5.5 \mathrm{M} €$, and $\mathrm{Y}_{3}: 6.5 \mathrm{M} €$, respectively) for WDM. For three years of suspension, the operator should invest $29 \mathrm{M} €$. In case of TDM, the total investment for the first four years is $26 \mathrm{M} €$, and with three years delay, it is $25 \mathrm{M} €$.

In the previous simulations, there was a gradually increasing rollout starting from $0 \%$ and achieving $100 \%$ at the final (8) year of the deployment. Alternative an operator can build the entire necessary infrastructure in the area in the first year of deployment (initial rollout at 100\%). A mediocre solution is to begin from $50 \%$ and achieve $100 \%$ at the end year. These three policies have been examined, and the results are presented in Fig. 13 for both TDM and WDM cases.

From Fig. 13, it can be deduced that in the case of $100 \%$ initial rollout, the cost in the first years is almost doubled and the prices converge after five years in each technology. Furthermore, it is interesting to note that in contrast with the previous obtained results, there are cases in which WDM is preferred against TDM. For example, the cost per user of WDM with initial deployment of $0 \%$ is lower than that of TDM with initial deployment of 50\% 


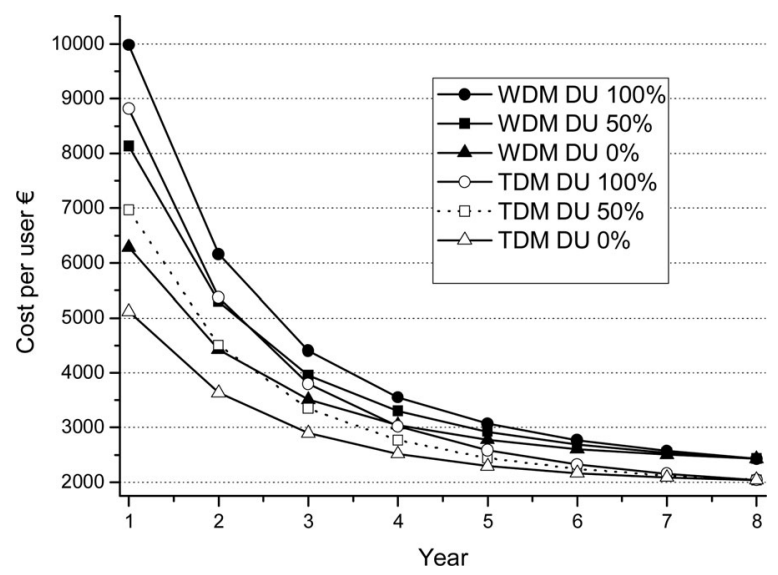

Fig. 13. Impact of different rollout policies.

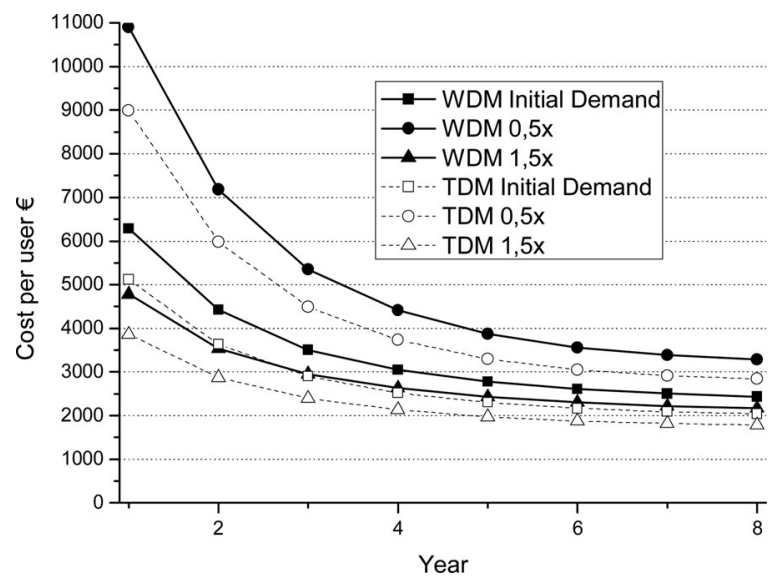

Fig. 14. Impact of demand uncertainty.

or $100 \%$. This is of great importance since it gives the operator the advantage of increased flexibility in his choices concerning both technology and rollout strategies.

One of the factors that greatly influence the deployment cost is the demand for broadband services. It should be noted that the demand forecast can be characterized by high uncertainty since it is a function of multiple parameters. Hence, one should extract a cost strip showing the lower and the upper limits corresponding to $0.5 x$ and $1.5 x$ of the initial forecasted values (see Fig. 14).

As shown in Fig. 14, the cost is highly sensitive to demand values. If the demand is half the initial value, the cost per user is almost doubled in the first years of network deployment. Hence, in order to facilitate network deployment, several stateaid measures such as demand stimulation/subsidization should be examined by national authorities.

\section{Dynamic and Emergent Behavior of the System-of-Systems Under Investigation}

In the previous sections, incremental cost methodologies along with different policies were implemented to evaluate the impact of unknown events such as deployment delay and different rollout strategies which are part of the evolution of the SoS. In order to further study the emergent behavior of the SoS under investigation, one should resort to techniques dealing with large

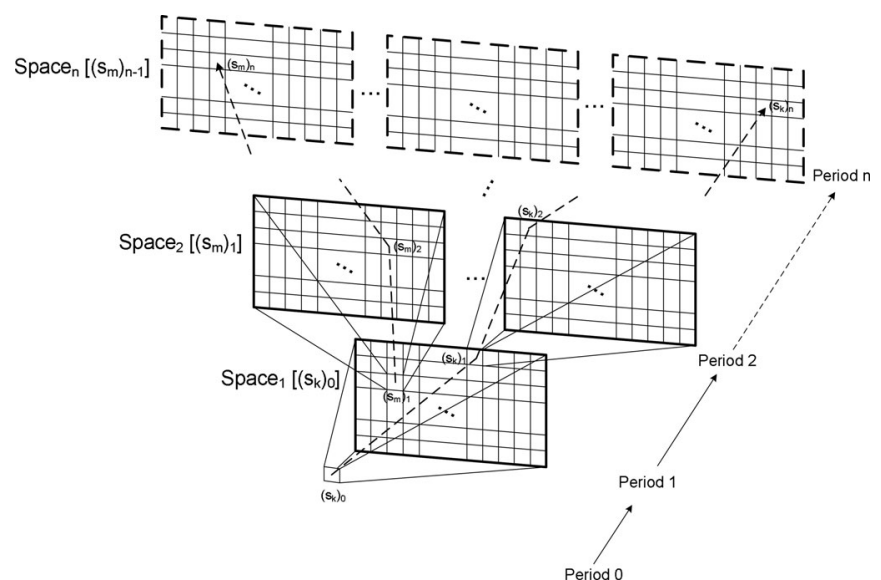

Fig. 15. Network realization along with uncertainty spaces.

degrees of uncertainty (deep uncertainty). Under conditions of deep uncertainty, it is hard to forecast the realizations and the time-varying relationships of relevant factors in the SoS. Furthermore, these situations of uncertainty can be occurred in a system that has not yet existed. Thus, new versions of the SoS can be obtained through the addition/deletion/replacement of systems or links. Unfortunately, in deep uncertainty, the appropriate conceptual models to describe interactions among SoS variables as well as the probability distributions to represent uncertainty about key parameters in the models are unknown.

In order to calm down model assumptions that are necessary to address uncertainty, a series of computational experiments should be performed. The numerical simulations include evaluations of model outcomes across a large set of possible SoS representations. Each plausible SoS representation can be assumed as one hypothesis about SoS behavior. By investigating a large set of such hypotheses and by evaluating their correctness, the "whole picture" of SoS emergent behavior can be obtained.

A simple method to construct the possible SoS models is to include time in the model specification defining the evolution of the SoS model. Without loss of generality, a discrete rather than continuous approach in modeling the time evolution is considered. The evolutionary modeling of SoS is depicted in Fig. 15.

As shown in Fig. 15, there is a dependence of uncertainty spaces in a period $i$ on the realizations of all the preceding periods. For example, in Period 2, the uncertainty space (Space ${ }_{2}$ $\left.\left[\left(s_{m}\right)_{1}\right]\right)$ is generated from the realization $\left(s_{m}\right)_{1}$ of the preceding uncertainty space (Space ${ }_{1}\left[\left(s_{k}\right)_{0}\right]$ ) which in turn is originated from the initial condition $\left(s_{k}\right)_{0}$. Hence, a possible future path (dashed lines) can be represented by the sequence $\left(s_{k}\right)_{0} \rightarrow$ $\left(s_{k}\right)_{1} \rightarrow\left(s_{k}\right)_{2} \ldots \rightarrow\left(s_{k}\right)_{n}$.

In this study, the time gap between two consequent periods is one year. For simplicity and since the provided results are just a representative example, the parameters involved in each uncertainty space (see Fig. 16) are limited to the penetration and the price of the optical related devices. Each combination between the penetration and the prices is a plausible SoS representation, while the path from the initial condition to this representation point describes the SoS behavior up to that period. It should be 


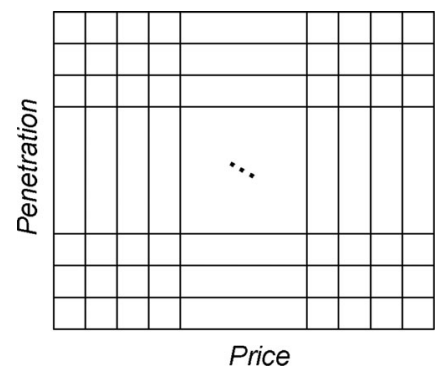

Fig. 16. SoS plausible representation for different combinations of penetration and price.
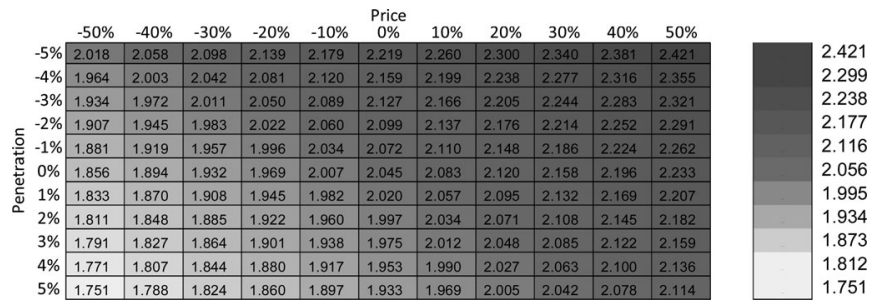

Fig. 17. One of the possible SoS representations after eight years in the case of TDM.
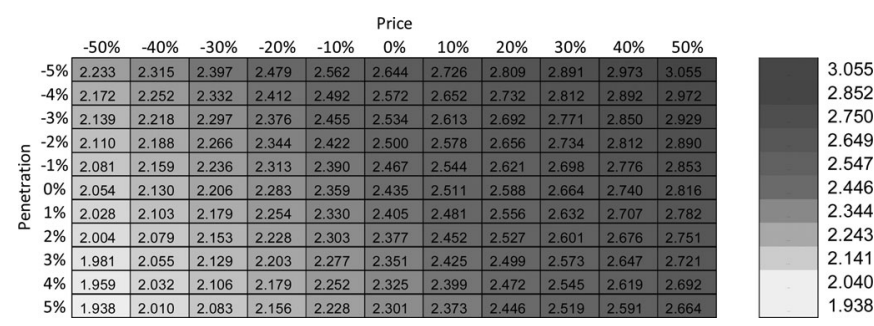

Fig. 18. One of the possible SoS representations after eight years in the case of WDM.

noted that the results obtained in previous sections correspond to specific paths of SoS exploratory representations.

In Figs. 17 and 18, one of the possible representations of SoS at the uncertainty space 7 (after eight years) is illustrated as a color map. Different colors correspond to different cost values per user in the case of TDM and WDM for the dense urban areas estimated using the methodologies described in previous sections. It should be highlighted that the penetration-price pairs used for the path up to this space is identical to that of Fig. 8.

Using the color map of Figs. 17 and 18, one can predict the impact of possible changes by estimating the cost values and determine the emergent behaviors of the SoS by exploring the corresponding paths.

In a next step, decision criteria can also be incorporated in order to determine whether a certain investment is economically possible according to expected budget and will, therefore, facilitate the investors. Hence, at the end of investment life (e.g., eight years), the performance of the investment must be superior (i.e., minimum cost). In this case, a criterion for a cost below the average cost of all $\mathrm{SoS}$ representations is assumed (see Figs. 19 and 20).

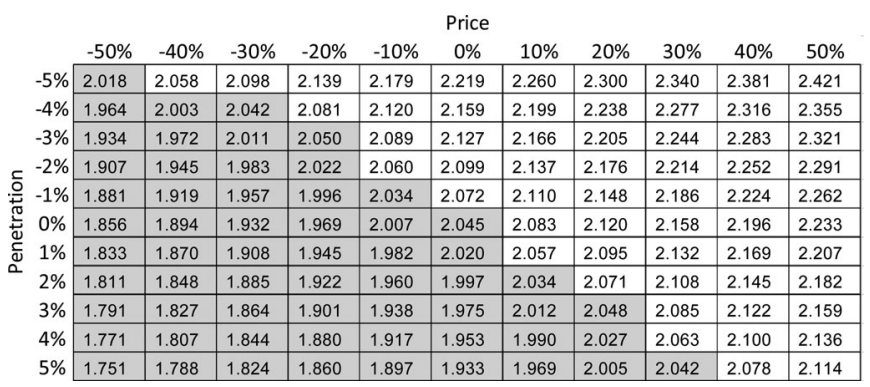

Fig. 19. Average cost as a decision criterion in the case of TDM.

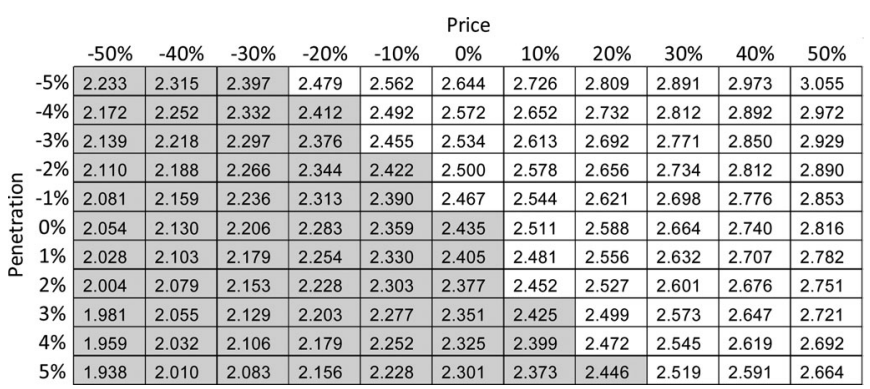

Fig. 20. Average cost per user as a decision criterion in the case of TDM.

\section{CONCLUSION}

In this paper, an SoS approach for telecommunications and especially FTTH networks has been introduced and illustrated. The characteristics of the SoS have been studied by investigating different adoption strategies for TDM and WDM technologies. The SoS evolution, that is, SoS changes stemming from link/system addition/replacement, was investigated through exploratory modeling. The installation costs of these networks in relation with several key parameters like geographical distribution and the year of introduction have been investigated and discussed. The cost per additional user follows the price curve shape, and it is of major importance for the decision process for a greenfield operator in order to estimate the maximum finance needed for the implementation. It should be also denoted that even the technology choice could be of minor importance for the incremental cost, at least from the SoS point of view, if the overall project is postponed for some years until the market and the main components are mature enough.

\section{ACKNOWLEDGMENT}

The authors would like to acknowledge the long-lasting fruitful collaboration with the partners of the IST-TONIC [31] and CELTIC-ECOSYS [42] technoeconomic projects, which offered methodologies, tools, and research ideas for the evaluation of telecommunications networks deployment strategies. They would also like to thank the associate editor and the anonymous reviewers for the fruitful comments which considerably improved this paper. 


\section{REFERENCES}

[1] A. Gorod, B. Sauser, and J. Boardman, "System-of-systems engineering management: A review of modern history and a path forward," IEEE Syst. J., vol. 2, no. 4, pp. 484-499, Dec. 2008.

[2] W. C. Baldwin and B. Sauser, "Modeling the characteristics of system of systems," in Proc. IEEE Int. Conf. Syst. Syst. Eng., May/Jun. 2009, pp. 1-6.

[3] SoS and FoS FAQ, Office of the Under Secretary of Defense for Acquisition Technology and Logistics.

[4] C. Keating, R. Rogers, R. Unal, D. Dryer, A. Sousa-Poza, R. Safford, W. Peterson, and G. Rabadi, "System of systems engineering," Eng. Manage. J., vol. 15, p. 36, Sep. 2003.

[5] P. Chen and J. Clothier, "Advancing systems engineering for systems-ofsystems challenges," vol. 6, pp. 170-183, 2003.

[6] A. Sousa-Poza, S. Kovacic, and C. Keating, "System of systems engineering: an emerging multidiscipline," Int. J. Syst. Syst. Eng., vol. 1, pp. 1-17, 2008.

[7] M. Mansouri, B. Sauser, and J. Boardman, "Applications of systems thinking for resilience study in maritime transportation system of systems," in Proc. 3rd Annu. IEEE Syst. Conf., 2009, pp. 211-217.

[8] N. Khabarov, E. Moltchanova, and M. Obersteiner, "Valuing weather observation systems for forest fire management," IEEE Syst. J., vol. 2 , no. 3, pp. 349-357, Sep. 2008.

[9] R. C. Byrd, J. M. Moss, W. C. Priedhorsky, C. A. Pura, G. W. Richter, K. J. Saeger, W. R. Scarlett, S. C. Scott, and R. L. Wagner, Jr., "Nuclear detection to prevent or defeat clandestine nuclear attack," IEEE Sensors J., vol. 5, no. 4, pp. 593-609, Aug. 2005.

[10] K. Daniel, B. Dusza, A. Lewandowski, and C. Wietfeld, "AirShield: A system-of-systems MUAV remote sensing architecture for disaster response," in Proc. 3rd Annu. IEEE Syst. Conf., 2009, pp. 196-200.

[11] I. Melgar, J. Fombellida, A. Jevtic, and J. Seijas, "Swarm architectures for ground-based air defense systems of systems," presented at the 7th IEEE Int. Conf. Ind. Informat., Cardiff, U.K., 2009.

[12] T. Ender, R. Leurck, B. Weaver, P. Miceli, W. D. Blair, P. West, and D. Mavris, "Systems-of-systems analysis of ballistic missile defense architecture effectiveness through surrogate modeling and simulation," in Proc. Annu. IEEE Syst. Conf., 2008, pp. 1-8.

[13] S. S. Durbha, R. L. King, and N. H. Younan, "An information semantics approach for knowledge management and interoperability for the global earth observation system of systems," IEEE Syst. J., vol. 2, no. 3, pp. 358365, Sep. 2008.

[14] Y. Hata, S. Kobashi, and H. Nakajima, "Human health care system of systems," IEEE Syst. J., vol. 3, no. 2, pp. 231-238, Jun. 2009.

[15] M. Jamshidi, Systems of Systems Engineering : Principles and Applications. Boca Raton, FL: CRC Press, 2009.

[16] T. Rokkas, D. Katsianis, and D. Varoutas, "Techno-economic evaluation of FTTC/VDSL and FTTH roll-out scenarios: Discounted cash flows and real option valuation," IEEE/OSA J. Opt. Commun. Netw., vol. 2, no. 9, pp. 760-772, Sep. 2010.

[17] T. Monath, N. K. Elnegaard, P. Cadro, D. Katsianis, and D. Varoutas, "Economics of fixed broadband access network strategies," IEEE Commun. Mag., vol. 41, no. 9, pp. 132-139, Sep. 2003.

[18] B. T. Olsen, D. Katsianis, D. Varoutas, K. Stordahl, J. Harno, N. K. Elnegaard, I. Welling, F. Loizillon, T. Monath, and P. Cadro, "Technoeconomic evaluation of the major telecommunication investment options for European players," IEEE Netw., vol. 20, no. 4, pp. 6-15, Jul./Aug. 2006.

[19] D. B. Agusdinata and D. DeLaurentis, "Specification of system-ofsystems for policymaking in the energy sector," Integr. Assess. J., vol. 8, pp. 1-24, 2008.

[20] S. Bankes, "Exploratory modeling for policy analysis," Oper. Res., vol. 41, pp. 435-449, 1993.

[21] M. Jamshidi, System of Systems Engineering: Innovations for the 21st Century. Hoboken, NJ: Wiley, 2009.

[22] Y. Ke-Wei, C. Ying-Wu, L. Yan-Jing, and Z. Qing-Song, "The study of guided emergent behavior in system of systems requirement analysis," in Proc. 5th Int. Conf. Syst. Syst. Eng., Jun. 2010, pp. 1-5.

[23] J. Gu and X. Tang, "Meta-synthesis approach to complex system modeling," Eur. J. Operational Res., vol. 166, pp. 597-614, 2005.

[24] C. D. Blair, J. T. Boardman, and B. J. Sauser, "Communicating strategic intent with systemigrams: Application to the network-enabled challenge," Syst. Eng., vol. 10, pp. 309-322, 2007.

[25] D. DeLaurentis and R. K. Callaway, "A system-of-systems perspective for public policy decisions," Rev. Policy Res., vol. 21, pp. 829-837, 2004.
[26] C.-H. Lee, W. V. Sorin, and B. Y. Kim, "Fiber to the home using a PON infrastructure," J. Lightw. Technol., vol. 24, no. 12, pp. 4568-4583, Dec. 2006.

[27] W. H. Melody, Telecom Reform: Principles, Policies and Regulatory Practices. Kongens Lyngby, Denmark: Tech. Univ. Denmark, 1997.

[28] EURESCOM-P901, European Institute for Research and Strategic Studies in Telecommunications GmbH, Heidelberg, Germany, 2001.

[29] C. M. Dippon and K. E. Train, "The cost of the local telecommunication network: A comparison of minimum spanning trees and the HAI model," Telecommun. Policy, vol. 24, pp. 253-262, 2000.

[30] L. Ims, Broadband Access Networks Introduction Strategies and Technoeconomic Evaluation. London, U.K.: Chapman \& Hall, 1998.

[31] TONIC/IST-25172, "Techno-economics of IP Optimised Networks and Services," 2000.

[32] R. D. Banker, H.-H. Chang, and S. K. Majumdar, "Economies of scope in the U.S. telecommunications industry," Inf. Econ. Policy, vol. 10, pp. 253 272, 1998.

[33] K. Stordahl and K. O. Kalhagen, "Broadband access forecasts for the European market," Telektronikk, vol. 98, pp. 21-32, 2002.

[34] C. Michalakelis, D. Varoutas, and T. Sphicopoulos, "Diffusion models of mobile telephony in Greece," Telecommun. Policy, vol. 32, pp. 234-245, Apr./May 2008.

[35] T. P. Wright, "Factors affecting the cost of airplanes," J. Aeronaut. Sci., vol. 3, pp. 122-128, 1936.

[36] A. B. Badiru and A. O. Ijaduola, "Half-life theory of learning curves for system performance analysis," IEEE Syst. J., vol. 3, no. 2, pp. 154-165, Jun. 2009.

[37] B. T. Olsen and K. Stordahl, "Models for forecasting cost evolution of components and technologies," Telektronikk, vol. 4, pp. 138-148, 2004.

[38] J. R. Crawford, Learning Curve, Ship Curve, Ratios, Related Data, Lockheed Aircraft Corp., Burbank, CA, 1944

[39] D. Katsianis, I. Welling, M. Ylonen, D. Varoutas, T. Sphicopoulos, N. K. Elnegaard, B. T. Olsen, and L. Budry, "The financial perspective of the mobile networks in Europe," IEEE Pers. Commun., vol. 8, no. 6, pp. 58-64, Dec. 2001.

[40] European Commission, "i2010 A European Information Society for Growth and Employment: Extended Impact Assessment," Brussels, Belgium: Commission of European Communities, (2005). [Online]. Available: http://ec.europa.eu/dgs/information_society/ evaluation/data/pdf/ia/i2010extended_impact_assessment.pdf

[41] Fiber to the Home Council Europe. [Online]. Available: http://www. ftthcouncil.eu/

[42] CELTIC-ECOSYS "Technoeconomics of Integrated Communication Systems and Services," (2002). [Online]. Available: http://www.celticinitiative.org/Projects/Celtic-projects/Call1/ECOSYS/ecosys-default.asp

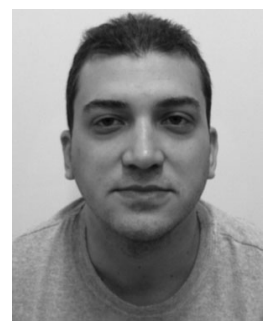

Theodore Rokkas received the B.Sc. degree in physics, the M.Sc. degree in electronics and radio communications, and the Ph.D. degree in technoeconomic evaluation of next-generation networks from the University of Athens, Athens, Greece.

$\mathrm{He}$ is currently with the University of Athens. He has actively participated in several technoeconomic activities for telecommunications, networks, and services, and in several European and national $R \& D$ projects. His research interests include technoeconomic evaluation of network architectures and services, mobile communication systems, broadband and wireless systems and networks, and methodologies for network design.

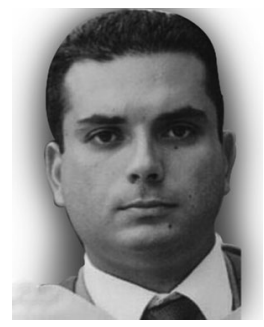

Ioannis Neokosmidis received the Physics degree, the M.Sc. degree in radioelectrology and electronics, and the Ph.D. diploma in optical nonlinear networks from the University of Athens, Athens, Greece.

He is currently a Research Associate and Adjunct Lecturer with the University of Athens. He participated in European and National projects. He has more than 30 publications with two best paper awards and more than 100 citations. His research interests include system of systems, deep uncertainties, optical communications, and technoeconomics. He serves as reviewer in leading IEEE/OSA Journals and conferences. His biography is listed in Marquis and Hübners Blaues Who's Who. 


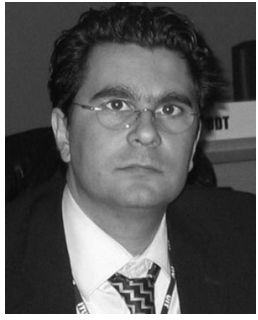

Dimitris Katsianis received the Informatics degree, the M.Sc. degree in signal processing and computational systems, and the Ph.D. diploma in network design with technoeconomics aspects from the Department of Informatics and Telecommunications, University of Athens, Athens, Greece.

$\mathrm{He}$ is a Senior Research Fellow with the Optical Communications Group, University of Athens, participating in several European and national R\&D projects. He has worked as an Expert Scientific Advisor with several firms in the field of technoeconomic and network design including national activities for technoeconomic evaluation of telecommunication strategies and regulation policies. He has more than 60 publications in journals and conferences in the field of technoeconomics and telecommunication network design.

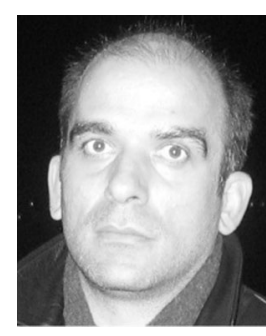

Dimitris Varoutas (M'98-SM'11) received the Physics degree, and the M.Sc. and Ph.D. degrees in communications and technoeconomics from the University of Athens, Athens, Greece.

$\mathrm{He}$ is currently an Assistant Professor with the Department of Informatics and Telecommunications, University of Athens. He has contributed to more than 100 publications in refereed journals and conferences in telecommunications, optoelectronics, and technoeconomics, including leading IEEE journals and conferences.

Dr. Varoutas is a Senior Member of the photonics (formerly LEOS), communications, education, and engineering management societies of the IEEE, and serves as a reviewer in several journals and conferences, including those of the IEEE. Since 2007, he has been a member of the BOG of ADAE, the National Authority for Communications Security and Privacy. 